\title{
Secondary Load-Frequency Control for MicroGrids in Islanded Operation
}

\author{
A. Madureira, C. Moreira and J. Peças Lopes \\ Faculdade de Engenharia da UP and Power Systems Unit of INESC Porto \\ Rua Dr. Roberto Frias, 378, 4200-465 Porto, Portugal \\ Phone:+351 22 2094000, Fax:+ 35122 2094050, E-mail: agm@inescporto.pt, cmoreira@inescporto.pt, jpl@fe.up.pt
}

\begin{abstract}
The objective of this paper is to present novel control strategies for MicroGrid operation, especially in islanded mode. The control strategies involve mainly the coordination of secondary load-frequency control by a MicroGrid Central Controller that heads a hierarchical control system able to assure stable and secure operation when the islanding of the MicroGrid occurs and in load-following situations in islanded mode.
\end{abstract}

\section{Keywords}

Renewable Energies, Dispersed Generation, Secondary Control

\section{Introduction}

The connection of microgeneration to Low Voltage (LV) networks is starting to deserve considerable attention from specialists worldwide, encouraging investigations and pilot experiences. In this context, a MicroGrid (MG) concept has been developed under the framework of the MicroGrids European Union project. A MG [1] can be defined as a LV distribution system to which small modular generation systems are connected. Generally, a MG corresponds to an association of electrical loads and small generation systems through a LV distribution network. This means that loads and sources are physically close.

Considering the currently available technologies, microgeneration systems may include several types of devices such as fuel-cells, wind turbines or photovoltaic (PV) systems as well as microturbines using either gas or bio-fuels.

Apart from a LV distribution network, microgenerators and electrical loads, a MG must also include some kind of storage devices (such as batteries or flywheels) as well as network control and management systems. The storage devices will play an important role in this kind of network, mainly in what concerns fast load-following situations.

At the current research status, it is assumed that the MG can be operated in two main situations:

- Normal Interconnected Mode - the MG will be electrically connected to the main MV network either being supplied by this network (totally or partially, depending on the generation allocation procedures adopted to operate the microsources) or injecting power into the main MV grid (when the relation between the microsources installed capacity and the electrical loads allows this type of operation);

- Emergency Mode - in case there is a failure in the main MV network, the MG must have the ability to operate in an isolated mode, that is, to operate in an autonomous way, similar to the power systems of physical islands.

A simulation platform under the MatLab® Simulink ${ }^{\circledR}$ environment was developed in order to evaluate the dynamic behaviour of several microsources operating together in a LV network under pre-specified conditions including interconnected and autonomous operation of the MG.

\section{Description of the Simulation Platform}

To test the effectiveness of the approach considered, a simulation platform under the MatLab® Simulink® environment was developed. At this stage only threephase balanced operation of the network is being considered.

The analysis requires the development of a set of dynamic models able to simulate the response of the MG under pre-specified conditions. For this purpose, several microsources and storage devices have been modelled. These models, together with control systems, were also implemented in the MatLab ${ }^{\circledR}$ Simulink ${ }^{\circledR}$ simulation platform. The models used are described in detail in [2].

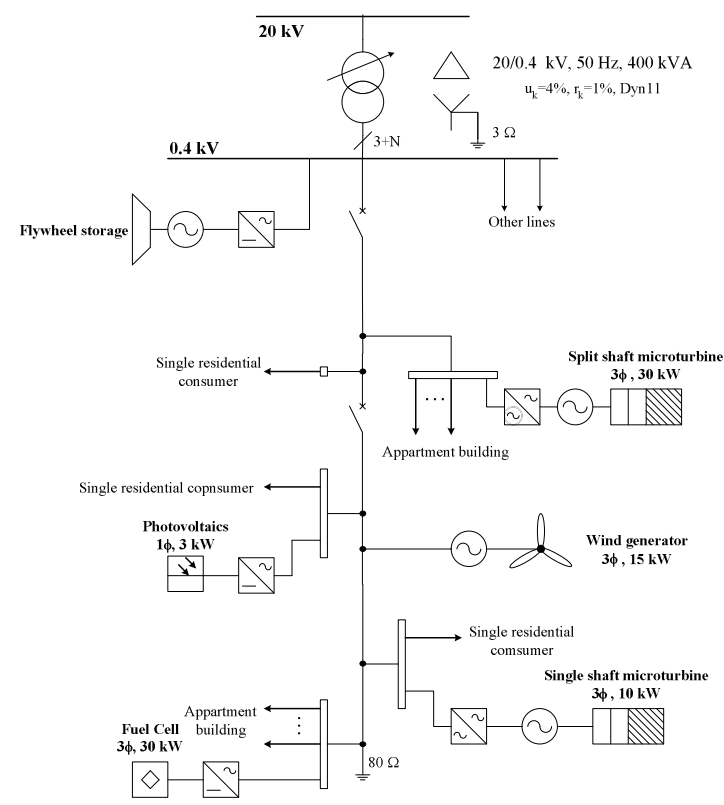

Fig. 1. LV NTUA test system 
Figure 1 illustrates the LV simulation test system developed by the NTUA [2] and adopted in this research. This network, together with its microsources and loads, was transposed to MatLab ${ }^{\circledR}$ Simulink ${ }^{\circledR}$ simulation platform, shown in Figure 2. It includes models and controls for microturbines (single-shaft and split-shaft), fuel-cells, small asynchronous wind generators, PV panels and storage devices (flywheels and batteries) as well as controllable loads (available for load-shedding purposes).

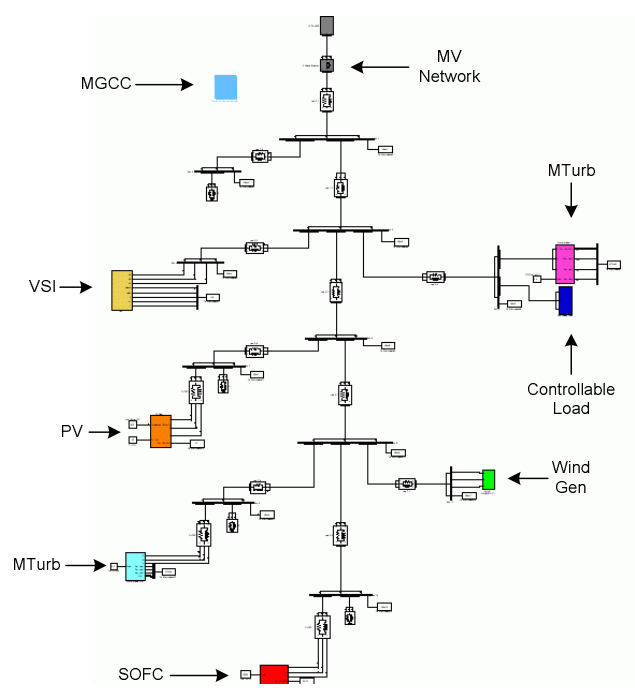

Fig. 2. Simulation platform under the MatLab® Simulink® environment

\section{MicroGrid Control Strategies}

\section{A. Main Concepts}

The main control strategy considered involves the passage to islanded operation mode of the MG in case of a fault in the MV network or in other exceptional cases. Contrary to the classic belief that islanded operation must be avoided at all costs, a new strategy is being developed that includes planned operation under these conditions. The islanding procedure is then controlled and made intentionally, corresponding to careful planning about operational conditions concerning not only load levels and levels of the distributed resources but also different types of defaults, etc.

The control of the MG is a delicate issue. A complex structure must be developed in order to assure a robust MG operation. The MicroGrid Central Controller (MGCC) is in charge of such MG operation control [3].

MG operation is based on a control scheme that exploits different inverter control modes [4]. This scheme requires frequency and voltage references that can be provided by a small diesel engine or by a Voltage Source Inverter (VSI). In this case one VSI connected to a flywheel device is used and the other inverters present act like current sources following the reference from the VSI or from the MV side, if available.

\section{B. MG Control and MGCC Structure}

The MGCC includes a multiplicity of functionalities one of which is secondary load-frequency control. This functionality is similar to the one of a conventional Automatic Generation Control (AGC) system. The MGCC coordinates a hierarchical control scheme, where the control infrastructure is shown in Figure 3.

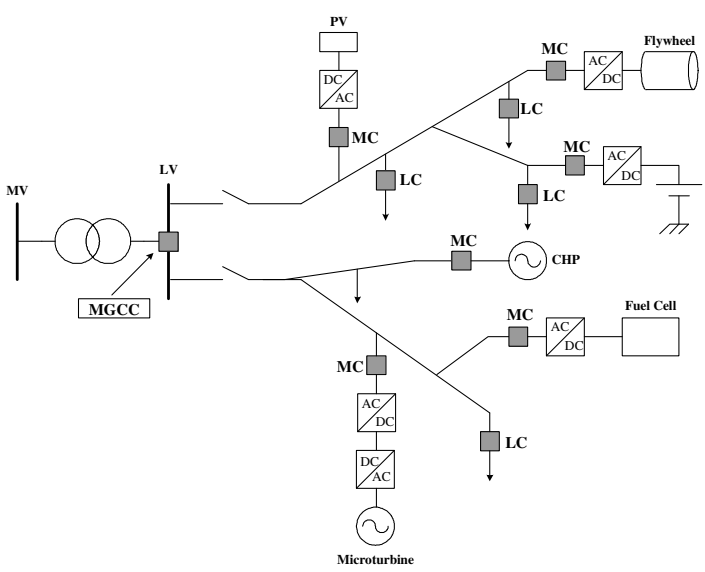

Fig. 3. MG control structure

The MGCC is supposed to be installed at the LV side of the MV/LV substation. The MGCC interfaces the MG and the distribution network and has several vital functions. At a second hierarchical level each microgenerator and storage device is locally controlled by a Microsource Controller (MC) and each electrical load is locally controlled by a Load Controller (LC). In order to achieve a good performance of the control scheme, an efficient communications infrastructure is necessary.

\section{Secondary Load-Frequency Control}

There are two ways of performing secondary loadfrequency control of the MG: either locally (using a local PI controller at each microsource, as represented in Figure 4) or in a centralized and automatic way, mastered by the MGCC. The load-frequency control is performed as follows: whenever the $\mathrm{MG}$ is operating in interconnected mode with the MV network, the centralized control is disabled; however, when the MG becomes isolated, the MGCC must coordinate the secondary load-frequency control.

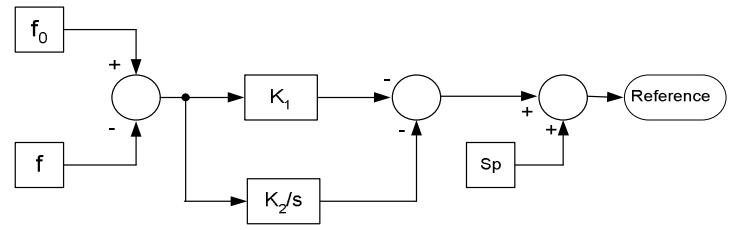

Fig. 4. Local secondary load-frequency control at each microsource 
The two main objectives of the secondary control at the MGCC are:

- To hold the system frequency at or very close to 50 $\mathrm{Hz}$;

- To maintain each unit's generation at the most economic value.

Notice that, unlike AGC standard implementation, tieline control is not applicable since only one control areathe $\mathrm{MG}$ - is considered.

The secondary load-frequency control is enabled subsequently to the action of local MCs in response to an imbalance between load and generation. This imbalance can be caused either by the islanding of the MG or by variations in load or in microsource generation levels [5] (like the ones that result from wind or PV generation).

In order to perform load-frequency control, the MGCC receives and stores information from the LCs (load levels) and MCs (microgeneration active power levels) and frequency measurements.

Using the frequency deviation as input and based on participation factors (pf) calculated using cost-functions associated with each microsource and economic setpoints for the microgenerators, the secondary frequency control function implemented at the MGCC specifies active power set-points that are sent back to the MCs in order to adjust the production levels and consequently correct the frequency offset.

The optimal economic set-point (Osp) for each microsource is updated every 60 seconds. These values are entered from a table that contains the results from economic dispatch for a market environment.

The centralized secondary load-frequency control structure is presented in Figure 5.

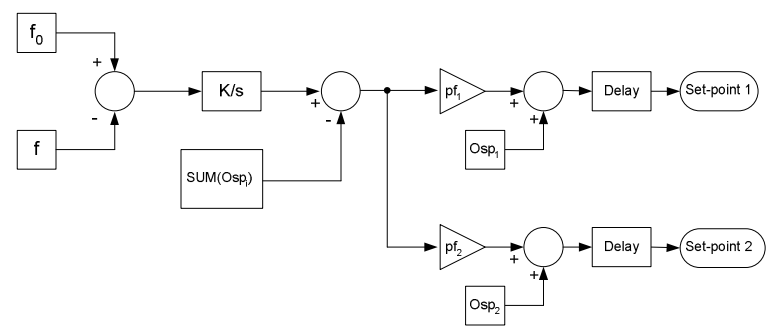

Fig. 5. Secondary load-frequency control at the MGCC

This particular structure uses only two microsources for secondary load-frequency control, but it can be generalized to include more microsources.

\section{MG Communication}

Communication issues in the MG deserve special attention due to the fast transients that may occur in the MG during its operation, resulting from its global low- inertia when in islanded mode. Because of this, inclusion of delays in the operation of the centralized secondary control scheme becomes a very important issue.

In order to account for the delays on the transmission of information either from the LCs and MCs to the MGCC and from the MGCC to the LCs and MCs, a block that represents both delays is included in the control scheme of the MG. On the other hand, to avoid unnecessary information circulation, the data on active power setpoints for microsources should not have a very small time period. Hence, the set-points are given by the MGCC to the MCs every 5 seconds.

Several options concerning the communications infrastructure are being investigated. Power Line Communication (PLC) is currently one of the most promising solutions but other solutions such as Wireless Access should not be excluded.

\section{Simulation Results}

Several simulations were performed in order to evaluate the dynamic behaviour of the MG involving the islanding of the MG, including load-following situations, with the centralized secondary load-frequency control approach developed. The NTUA test system was used for the evaluation of the effectiveness of these strategies. Results using local control and centralized control by the MGCC are compared in this section.

The dynamic behaviour of the MG was evaluated after a disconnection of the MV network at $\mathrm{t}=40 \mathrm{~s}$. The initial total load of the MG was around $72 \mathrm{~kW}$ and the generation from the microsources (before the islanding of the MG) was around $32 \mathrm{~kW}$. In face of the observed frequency deviation, some load was shedded in order to aid frequency restoration. The load was later reconnected in small load steps, allowing the evaluation of $\mathrm{MG}$ behaviour under load-following conditions.

\section{A. Local Control}

Results obtained with the local PI frequency control approach are presented in the next two figures. The MG frequency and the active power output response of the SOFC and of the microturbine are depicted.

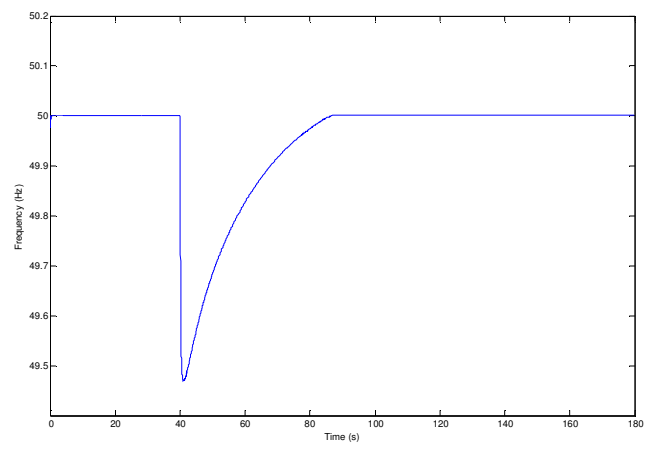

Fig. 6. Frequency of the MG 


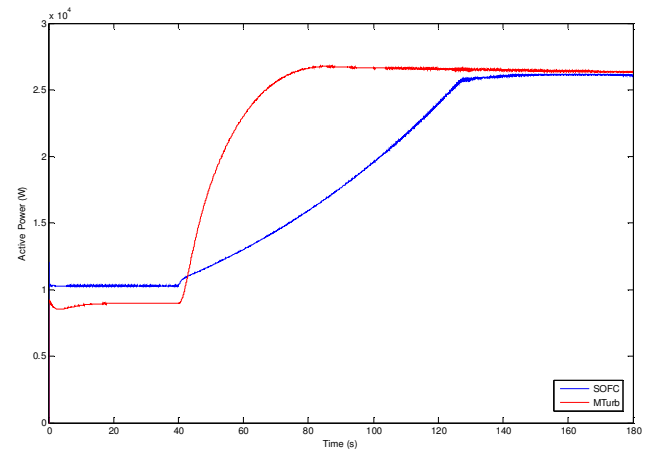

Fig. 7. Active power in the SOFC and the microturbine

\section{B. Centralized Control}

Among the microsources present at the MG, the SOFC and the single-shaft microturbine are the more suitable ones for performing secondary control, since the primary energy source is available and not depending on intermittency as it happens with the wind generator or the PV panel. These two microgenerators guarantee the secondary load-frequency control, receiving orders from the MGCC via the corresponding MC.

Two sets of results are presented. The first considers constant wind and irradiance feeding the wind generator and the PV. The second considers random variations in both wind velocity and irradiance.

The main results obtained with constant wind speed and irradiance are presented in the following figures.

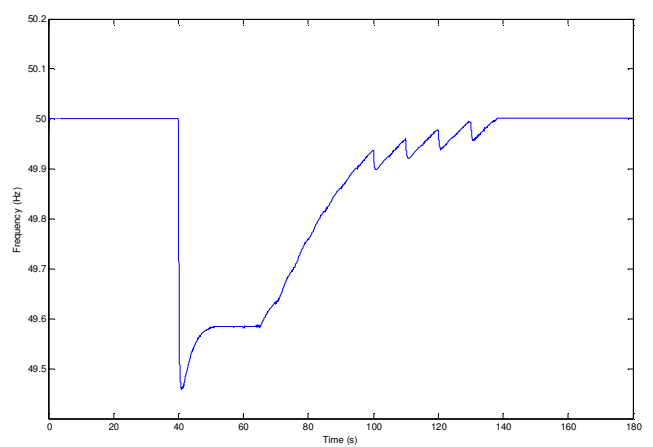

Fig. 8. Frequency of the MG

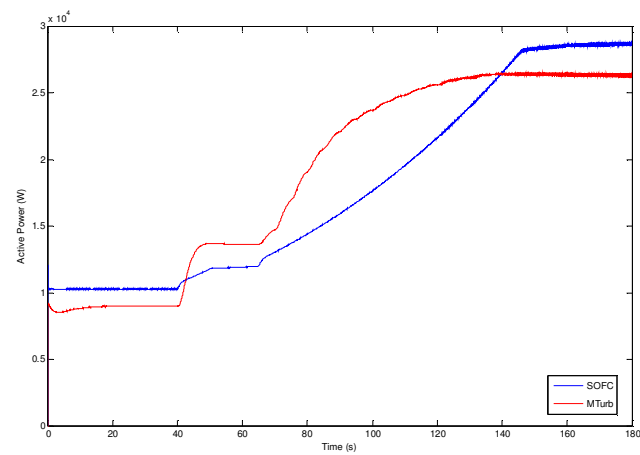

Fig. 9. Active power in the SOFC and the microturbine
When comparing this response with the one obtained using local frequency control it can be noticed that centralized control is slightly slower.

The main results obtained with variable wind speed and irradiance are presented in the next two figures.

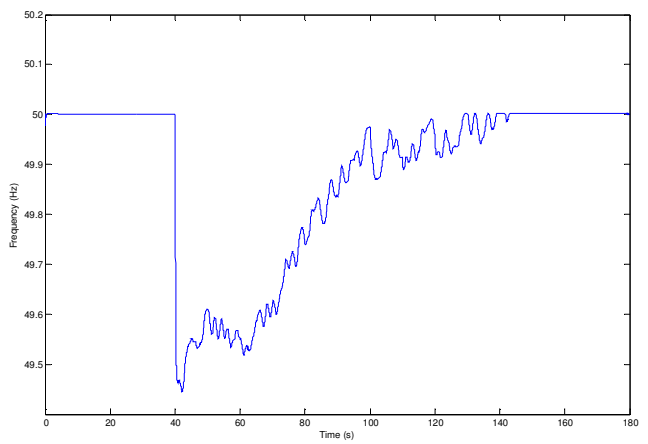

Fig. 10. Frequency of the MG

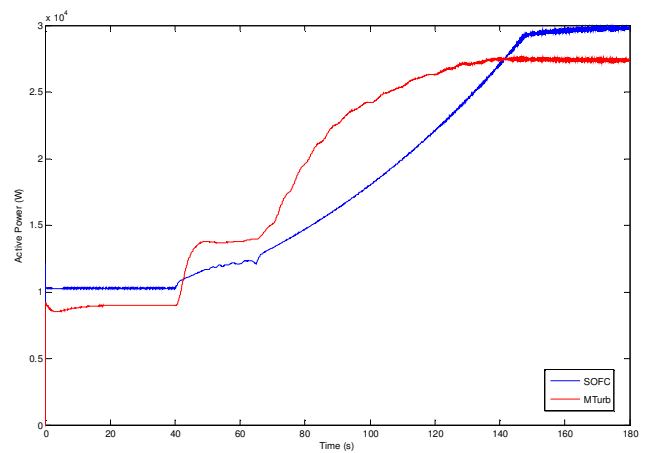

Fig. 11. Active power in the SOFC and the microturbine

It can be seen that frequency in islanded mode has several oscillations due to variations in wind speed and irradiation.

\section{Conclusion}

Simulation results suggest that the islanding of the MG can be performed safely under various operating conditions and that the MGCC assures both secure and stable operation in islanded mode.

It has been shown that centralized secondary control exhibits a good performance using the secondary loadfrequency control algorithm presented at the MGCC, when compared to the reference case of local secondary control. In addition, centralized secondary loadfrequency control allows the management of the MG according to both technical and economical criteria.

\section{Acknowledgement}

The authors thank the European Commission for financial support within the framework of EU Project MicroGrids, Contract No. ENK-CT-2002-00610. 


\section{References}

[1] R. Lasseter et al, "The CERTS Microgrid Concept”, White paper on Integration of Distributed Energy Resources, April 2002.

[2] N. Hatziargyriou et al, "Modelling of Micro-Sources for Security Studies", CIGRE Session, 2004.

[3] J. Peças Lopes, J. Tomé Saraiva, N. Hatziargyriou, N. Jenkins, "Management of MicroGrids", JIEEC2003 Bilbao, 2003.

[4] S. Barsali, M. Ceraolo, P. Pelacchi, "Control techniques of Dispersed Generators to improve the continuity of electricity supply", IEEE, 2002.

[5] B. Delfino, F. Fornari, S. Massuco, "Load-Frequency Control: new perspectives in the open access environment", IEEE, 2000.

[6] J. Peças Lopes, C. Moreira, A. Madureira, "Emergency Strategies and Algorithms", MicroGrids project deliverable DD1, October 2004. 